Comparative Assessment of Topical Glucantime ${ }^{\circledR}$ Injection Plus Cryotherapy and Cryotherapy Alone for Treatment of Anthroponotic

\title{
Cutaneous Leishmaniasis
}

\author{
Mohammad Reza Shirzadi ${ }^{1}$, Amir Hamta $\mathbb{( D}^{2}$, Leyli Zanjirani Farahani ${ }^{3}$, Abedin Saghafipour (D) ${ }^{4}{ }^{*}$ and \\ Sadegh Rahi ${ }^{5}$ \\ ${ }^{1}$ Center for Communicable Diseases Management, Ministry of Health and Medical Education, Tehran, Iran \\ ${ }^{2}$ Department of Biostatistics School of Medicine Arak University of Medical Sciences, Arak, Iran \\ ${ }^{3}$ Department of Medical Parasitology and Mycology, School of Public Health, Tehran University of Medical Sciences, Tehran, Iran \\ ${ }^{4}$ Department of Public Health, Faculty of Health, Qom University of Medical Sciences, Qom, Iran \\ ${ }^{5}$ Bam Provincial Health Center, Bam University of Medical Sciences, Bam, Iran \\ "Corresponding author: Department of Public Health, Faculty of Health, Qom University of Medical Sciences, Qom, Iran. Email: abed.saghafi@yahoo.com
}

Received 2020 December 16; Revised 2021 July 25; Accepted 2021 August 15.

\begin{abstract}
Background: Cutaneous leishmaniasis is a worldwide health problem due to the complications in the transmission cycle, treatment failure, and post-healing problems.

Objectives: Infected humans are the parasite reservoirs in the transmission cycle of anthroponotic cutaneous leishmaniasis (ACL); thus, the lack of efficient treatment increases the risk of the disease outbreak. The gold standard treatment for CL is mainly the topical or systematic use of antimonial compounds. Much research has been done on the development of topical therapy alone or in combination with cryotherapy. Therefore, this study was conducted to comparatively evaluate the current treatment protocol, cryotherapy, versus combined cryotherapy (Cryo) and intralesional Glucantime ${ }^{\circledR}$ (IG).

Methods: This quasi-experimental study was performed on 476 patients with cutaneous leishmaniasis lesions referred to the health centers of a CL endemic area in southern Iran (Bam County, Kerman Province) during 2013 - 2019. A total of 200 cases with 301 lesions received Cryo using liquid nitrogen once every two weeks, and 276 cases with 406 lesions received weekly IG combined with Cryo, once every two weeks. The lesion size was recorded before and after the treatment. At seven and 12 weeks after the treatment, the lesion healing rate was monitored. Healing was defined as complete re-epithelialization. The obtained data were assessed using logistic regression to calculate the relative risk and therapeutic effects.

Results: Out of 476 CL patients with 709 lesions, 200 cases with 221 lesions received Cryo, and 276 patients with 292 lesions received Cryo plus IG. At the seventh week after the treatment, complete healing was observed in 100 out of 221 cases (50\%) in the group that received biweekly Cryo only and 225 out of 276 cases (81.5\%) in the group that received biweekly Cryo plus weekly IG $(P=0.001)$. Conclusions: This study found that the use of cryotherapy plus intralesional Glucantime ${ }^{\circledR}$ increases the chance of healing for patients by 4.4 times compared to the use of cryotherapy alone.
\end{abstract}

Keywords: Glucantime ${ }^{\circledR}$, Anthroponotic Cutaneous Leishmaniasis, Iran

\section{Background}

Cutaneous leishmaniasis (CL) is a chronic parasitic skin disease that is transmitted from animal or human reservoirs to humans through the bite of the female sandfly. About 700,000 to one million new cases of CL are reported annually in more than 102 endemic countries worldwide (1). The infection not only is usually associated with mortality but also causes skin lesions, which persist for months or sometimes for years. Skin lesions often follow a thick scar, even after healing with standard treat- ment. Moreover, secondary bacterial and fungal contamination in the lesion might cause other problems, including septicemia and even tetanus, which makes the treatment of the disease more significant. Self-healing zoonotic CL (ZCL) caused by Leishmania major and anthroponotic CL (ACL) caused by L. tropica are two common types of CL. The main reservoir of the parasite in ACL is the human (2). Currently, ZCL is endemic in 17 provinces, and ACL is common in many areas of IR. Iran, including Bam city in Kerman province, southeastern Iran. The incidence of CL (zoonotic 
and anthroponotic) in Iran in recent years has amounted to an average of 30 cases per 100,000 inhabitants, and the average number of cases is approximately 20,000 per year, while the actual number of cases is estimated to be four to five times higher than the number of cases reported $(3,4)$. The condition of the disease cycle and the lack of an effective vaccine could hinder the effectiveness of disease prevention methods. Based on studies in the city of Bam, it has been concluded that the timely diagnosis and treatment of patients play an important role in the control of the disease $(5,6)$.

The most definitive method of diagnosing CL includes two methods, including direct smear preparation and culture of leech wound secretions to observe the stages of the Leishmania amastigote and promastigote parasite. Both methods are certainly valuable, although the cultivation method takes a long time. These methods have the advantage of simplicity, but they are less sensitive to species and do not detect them. Polymerase chain reaction (PCR) is a reliable method with more sensitivity than conventional methods or clinical and epidemiological features (3).

Currently, antimony compounds including Glucantime $^{\circledR}$ (meglumine antimoniate) and pentostam (sodium stibogluconate) are used in many countries for the treatment of CL patients, with systemic or intralesional injection. Cryotherapy using nitrogen dioxide or $\mathrm{CO}_{2}$ is also used in patients with side effects. According to the guidelines of the Ministry of Health of Iran, in the cases of CL lesions on patients' faces, the lesions with more than $3 \mathrm{~cm}$ diameter, sporotrichoid forms, the lesions on the joints skin, and recurrent cases that have more rates of treatment failure, systemic Glucantime ${ }^{\circledR}$ injection is preferable; otherwise, topical treatments are advised. The standard method for topical treatment includes the intralesional injection of Glucantime ${ }^{\circledR}$ once a week and cryotherapy once every two weeks until complete healing of the lesion or for up to 12 weeks. In addition, in patients with CL who have underlying diseases such as myocarditis, hepatitis, pancreatitis, or renal failure and also in pregnant or lactating women, Glucantime ${ }^{\circledR}$ is not recommended; so cryotherapy is prescribed solely (7). While choosing a treatment method, the prevention of drug resistance besides side effects and availability of the medication should be considered, along with economic efficiency (8). Furthermore, the appropriate treatment should be selected considering the type of the disease, ACL or ZCL, and the location, number, size, type, and distribution of the lesions. The results of previous studies suggest that the small lesions of ZCL may heal more spontaneously, even if not treated, but in the case of ACL, in which patients are the main reservoirs of the parasite, the need for efficient and timely treatment of skin lesions has been emphasized ( 9 ).

\section{Objectives}

The conventional gold standard treatment for CL is mainly intralesional or systematic administration of antimonial compounds. Lately, several investigations have been made on the development of a topical treatment based on single or combined therapies. It is important to provide a relatively economic and effective treatment for CL patients because of undesirable consequences of the disease to reduce the duration of treatment and prevent drug resistance. Furthermore, the early treatment of ACL is important in the control of the disease spread in endemic areas. Therefore, this study was conducted to evaluate the effectiveness of cryotherapy alone and cryotherapy plus intralesional injection of Glucantime ${ }^{\circledR}$ in the treatment of ACL in one of the main endemic regions of ACL, Bam, Kerman Province, southeast Iran.

\section{Methods}

This quasi-experimental study was performed on 476 patients with ACL during the years 2013 to 2019, in Bam City of Kerman Province, located in southeastern Iran, as the most infected endemic area of ACL. Definitive diagnosis of CL was made by direct smear and observation of LeishmanDonovan bodies containing parasites in the samples prepared from skin lesions. Finally, the PCR technique was used to confirm the diagnosis of parasite species.

All patients referred to health centers in Bam during 2013 - 2019 entered the study. Criteria for entering the study included a diagnosis of leishmaniasis based on the observation of Leishman bodies in smears from skin lesions, the age over one-year-old, and the onset of the disease in less than four weeks. The exclusion criteria from the study included the use of antibiotics or other antimicrobial drugs in the past four weeks, lactating and pregnant mothers, having extensive lesions with a diameter more than $5 \mathrm{~cm}$, and more than three lesions on the patient's body. For all participants or their parents, a written consent form was filled, according to the principles of medical ethics. This study met the World Health Organization definition of a clinical trial with registration number IRCT20170723035248N4. Also, the authors confirm that 
all ongoing and related trials for this drug intervention are registered.

Patients were divided into two groups randomly. Of 476 patients who completed the study, 276 used cryotherapy plus intralesional Glucantime ${ }^{\circledR}$ injection, and 200 patients received cryotherapy alone (Figure 1). In the first group, Glucantime ${ }^{\circledR}$ (Specia, Paris, France) with a concentration of 1.5 grams per $5 \mathrm{~mL}$, at a dose of $0.5 \mathrm{~mL}$ to $2 \mathrm{~mL}$ depending on the lesion size, was injected using insulin syringe needles in adjacent intact skin of the lesions until the margin of the lesion became completely white. The injection was repeated once a week for up to 12 weeks. Also, in this group, cryotherapy was administered every two weeks, before the IG injection, up to six times simultaneously. In the second group, cryotherapy was administered every two weeks, up to six times. For cryotherapy, liquid nitrogen was applied to the lesion using a sterilized cotton swab until a $2-3 \mathrm{~mm}$ halo formed around it for 10 30 seconds.

After the 12th injection or sixth cryotherapy session, all patients in both groups were examined. Healing was considered as complete re-epithelialization and disappearance of induration. A follow-up was done for patients up to six months. The elimination of inflammatory symptoms and tissue hardness, and healing of papules and ulcers were considered as complete recovery. In addition, in patients with more than one lesion, all lesions would be healed to be considered as cured. All untreated cases were referred to medical centers for more evaluations and other treatment protocols.

The data were compiled as a standard epidemiological study, approved by the Center of Communicable Disease Management of the Ministry of Health in Iran. To calculate the raw and adapted odds ratios of the efficacy of cryotherapy alone compared to cryotherapy plus intralesional injection of Glucantime ${ }^{\circledR}$ for CL treatment, single-variable and multivariate logistic regression models were used. The significance level was considered as $\mathrm{P}<0.05$.

\section{Results}

Of 476 patients with 709 lesions of Anthroponotic $\mathrm{Cu}-$ taneous leishmaniasis (ACL) who completed the study, 319 (67\%) were females, and 157 (33\%) were males. The age range of the patients was 1 to 92 years, with an average age of 32.8 years, with the majority of the patients $(n=374,78.6 \%)$ being in the age group over 15 years. Besides, $64 \%$ of the lesions were on the hands, $21 \%$ on the face, $19 \%$ on the feet, and the rest on the other parts of the body. The specifica- tions of the study groups, including age, gender, nationality, place of residence (city or village), location of the lesion, size of the lesion in centimeters, and the number of lesions, are shown in Table 1 . Totally, $85.5 \%$ and 50\% of the patients with ACL were treated in the two groups of cryotherapy plus intralesional injection of Glucantime ${ }^{\circledR}$ and cryotherapy alone, respectively (Table 2 ).

On average, the probability of recovery was $4.412(2.9$ - 6.6) times higher in people who received intralesional Glucantime ${ }^{\circledR}$ combined with cryotherapy than in those who received cryotherapy alone $(\mathrm{P}<0.001)$.

\section{Discussion}

Choosing a proper treatment for cutaneous leishmaniasis has been a health challenge in the endemic areas for many years. Leishmaniasis is endemic in several parts of Iran. Antimonial compounds are used as the first-line treatment, which has caused drug resistance due to intermittent use recently. The effectiveness of this method varies from $68 \%$ to $82 \%$ based on different studies $(10,11)$. The reason for the different results with this treatment may be related to the characteristics of the studied population, lesion condition, the difference in parasite species, and drug resistance $(12,13)$. Cryotherapy is an effective non-pharmacological treatment for leishmaniasis with no systemic side effects since Leishmania sp. is potentially thermo-sensitive. This method usually requires multiple sessions in about 12 weeks, with treatment failure rates up to $50 \%$ and relapse rates up to $8 \%$. Antimonial compounds are associated with significant side effects but have lower failure rates. Several studies have compared intralesional Glucantime with cryotherapy either alone or as a combined therapy (14).

In a study conducted in Ethiopia, the treatment response of CL to cryotherapy using liquid nitrogen and intralesional injection of generic sodium stibogluconate was evaluated in 103 patients. The cure rate for cryotherapy was 93\%, and the major adverse events were blister formation immediately after the application of liquid nitrogen, erythema, and burning sensation. Of the total patients who received SSG, $85 \%$ were cured, and $10 \%$ were unresponsive. Patients who were not cured by intramuscular SSG were treated with combination therapy (intralesional SSG injection and cryotherapy). The cure rate for cryotherapy and Pentostam was obtained as $93.3 \%$ and $89.5 \%$, respectively. Using liquid nitrogen for cryotherapy to treat dermal leishmaniasis is more convenient and easier than the application of other methods $(15,16)$. In our study, the same re- 


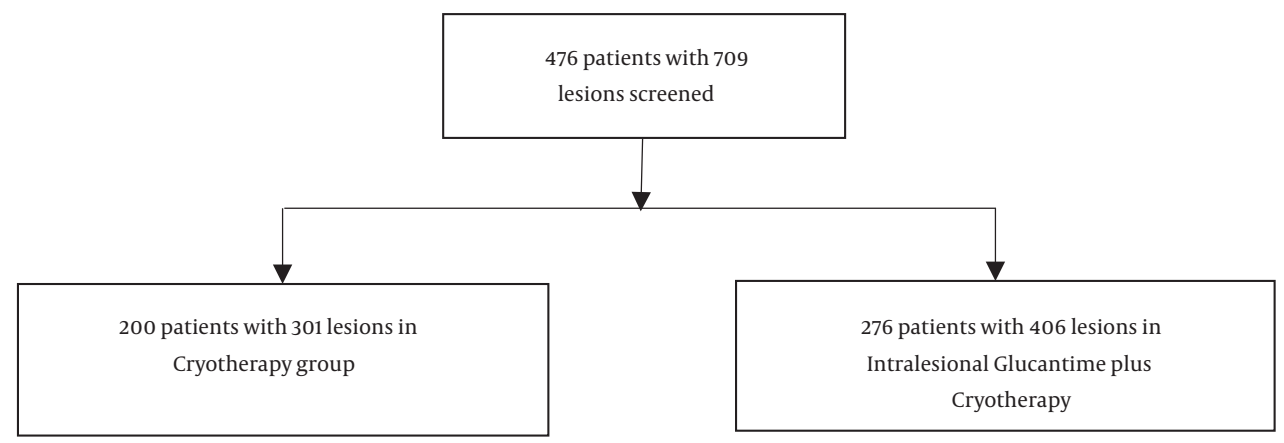

Figure 1. A total of 476 patients completed follow-up after two types of treatment for investigating cryotherapy alone versus cryotherapy plus Intralesional Glucantime ${ }^{\circledR}$ therapeutic efficacy in anthroponotic cutaneous leishmaniasis, Bam County, Kerman Province, 2013 - 2019.

Table 1. Some Demographic and Epidemiologic Characteristics of Patients Participating in the Study of Evaluating Current Treatment Protocols for Anthroponotic Cutaneous Leishmaniasis in Bam County, Kerman Province, 2013 - 2019

\begin{tabular}{|c|c|c|}
\hline \multirow{2}{*}{ Characteristics } & \multicolumn{2}{|c|}{ Types of Treatment } \\
\hline & Number (\%) of Patients Who Received Cryotherapy Alone & $\begin{array}{c}\text { Number }(\%) \text { of Patients Who Received Intralesional Glucantime }{ }^{\circledR}+ \\
\text { Cryotherapy }\end{array}$ \\
\hline \multicolumn{3}{|l|}{ Gender } \\
\hline Male & $27(13.5)$ & $130(47.1)$ \\
\hline Female & $173(86.5)$ & $146(52.9)$ \\
\hline \multicolumn{3}{|l|}{ Age } \\
\hline$<1$ & $0.00(0.00)$ & $0.00(0.00)$ \\
\hline $1-5$ & $2(1.00)$ & $19(6.9)$ \\
\hline $5-15$ & $6(3)$ & $75(27.2)$ \\
\hline$>15$ & $192(96)$ & $182(65.9)$ \\
\hline \multicolumn{3}{|l|}{ Nationality } \\
\hline Iranian & $199(99.5)$ & $271(98.2)$ \\
\hline Afghan & $1(0.5)$ & $5(1.8)$ \\
\hline \multicolumn{3}{|l|}{ Location of lesions } \\
\hline Hand & $137(68.5)$ & $170(61.6)$ \\
\hline Foot & $36(18)$ & $57(20.7)$ \\
\hline Face & $44(22)$ & $58(21)$ \\
\hline Head and neck & $0(0.00)$ & $5(1.8)$ \\
\hline \multicolumn{3}{|l|}{ Number of lesions (\%) } \\
\hline Trunk & $4(2)$ & $2(0.7)$ \\
\hline 1 & $140(70)$ & $196(71)$ \\
\hline 2 & $35(17.5)$ & $49(17.8)$ \\
\hline 3 & $14(7)$ & $18(6.5)$ \\
\hline 4 & $3(1.5)$ & $8(2.9)$ \\
\hline$>4$ & $8(4)$ & $5(1.8)$ \\
\hline \multicolumn{3}{|l|}{ Type of lesions } \\
\hline Nodule & $66(33)$ & $81(29.35)$ \\
\hline Papule & $115(57.5)$ & $152(55.07)$ \\
\hline Ulcer & $19(9.5)$ & $43(15.58)$ \\
\hline \multicolumn{3}{|l|}{ Size of lesions, cm } \\
\hline 1 & $122(61)$ & $186(67.4)$ \\
\hline 2 & $39(19.5)$ & $56(20.3)$ \\
\hline 3 & $28(14)$ & $25(9.1)$ \\
\hline$>3$ & $11(5.5)$ & $9(3.3)$ \\
\hline \multicolumn{3}{|l|}{ Residency status } \\
\hline Rural areas & $80(40)$ & $163(59.1)$ \\
\hline Urban areas & $120(60)$ & $113(40.9)$ \\
\hline
\end{tabular}

sult was obtained, and the complete cure of ACL lesions was seen in $81.5 \%$ of the cases taking both Cryo and IG treat- ments.

One of the major causes of failure in CL treatment 
Table 2. Cure Rates of Current Treatment Protocols for Anthroponotic Cutaneous Leishmaniasis in Bam County, Kerman Province, 2013 - 2019

\begin{tabular}{lccc}
\hline Cure Rate & \multicolumn{3}{c}{ Treatments } \\
\cline { 2 - 4 } & $\begin{array}{c}\text { Cryotherapy, } \\
\text { No. (\%) }\end{array}$ & $\begin{array}{c}\text { Intralesional } \\
\text { Glucantime } \\
\text { Plus } \\
\text { Cryotherapy, } \\
\text { No. (\%) }\end{array}$ & Total, No. (\%) \\
\hline Cured & $100(50)$ & $225(81.5)$ & $325(68.3)$ \\
Not cured & $100(50)$ & $51(18.5)$ & $151(31.7)$ \\
\hline Total & $200(100)$ & $276(100)$ & $476(100)$ \\
\hline
\end{tabular}

with intralesional injection of Glucantime ${ }^{\circledR}$ is poor drug infiltration into the lesion. Also, the placement of Leishmania parasites in parasitophorous vacuoles within macrophages, which is a way to escape from the patient's immune system, makes the parasite resistant to pharmacological treatment. Cryotherapy makes the parasites more exposed to the immune system and topical drugs by tearing up the membrane of macrophages, which improves the healing process $(17,18)$. This effect was well seen in the present study. Therefore, the chance of complete recovery in patients who simultaneously received both treatments was higher than those receiving the single method of treatment.

\subsection{Conclusions}

Based on the results of the present study, the combined use of two methods of intralesional injection of Glucantime ${ }^{\circledR}$ and cryotherapy using liquid nitrogen for the treatment of ACL can reduce the treatment duration and increase the chance of complete recovery in the patients.

\section{Acknowledgments}

We would like to thank Dr. Jose Antonio Ruiz-Postigo, from the Department of Control of Neglected Tropical Diseases, at the World Health Organization in Geneva, Switzerland, for his valuable comments and critical advice.

\section{Footnotes}

Authors' Contribution: Abedin Saghafipour and Mohammad Reza Shirzadi conceived and co-designed the study. Abedin Saghafipour, Amir Hamta, and Leyli Zanjirani Farahani analyzed and interpreted the data. Anedin Saghafipour, Mohammad Reza Shirzadi, Leyli Zanjirani Farahani, and Sadegh Rahi provided reagents, materials, analysis tools, or data. Abedin Saghafipour and Sadegh Rahi collected the primary data. All authors contributed to the writing of the manuscript.

Clinical Trial Registration Code: The clinical trial registration code was IRCT20170723035248N4.

Conflict of Interests: The authors declare no conflict of interest.

Ethical Approval: This study was approved by the Ethics Committee of Qom University of Medical Sciences.

Funding/Support: Funding for this research was provided by the Research and Technology Center of Qom University of Medical Sciences.

Informed Consent: Written informed consent was obtained from participants.

\section{References}

1. Ruiz-Postigo JA, Grout L, Saurabh J. Global leishmaniasis surveillance, 2017-2018, and first report on 5 additional indicators/Surveillance mondiale de la leishmaniose, 2017-2018, et premier rapport sur 5 indicateurs supplementaires. Week Epidemiol Record. 2020;95(25):265-80.

2. Salimi M, Jesri N, Javanbakht M, Farahani LZ, Shirzadi MR, Saghafipour A. Spatio-temporal distribution analysis of zoonotic cutaneous leishmaniasis in Qom Province, Iran. J Parasit Dis. 2018;42(4):570-6. doi: 10.1007/s12639-018-1036-5. [PubMed: 30538355]. [PubMed Central: PMC6261142].

3. Piroozi B, Moradi G, Alinia C, Mohamadi P, Gouya MM, Nabavi M, et al. Incidence, burden, and trend of cutaneous leishmaniasis over four decades in Iran. Iranian J Public Health. 2019;48(Supple 1):28-35.

4. Saghafipour A, Moradi-Asl E, Rassi Y, Hanafi-Bojd A. Spatial distribution and infection rate of leishmaniasis vectors (Diptera: Psychodidae) in Ardabil Province, Northwest of Iran. Asian Pac J Trop Biomed. 2019;9(5). doi: 10.4103/2221-1691.258997.

5. Saghafipour A, Vatandoost H,Zahraei-Ramazani AR, Yaghoobi-Ershadi MR, Jooshin MK, Rassi Y, et al. Epidemiological Study on Cutaneous Leishmaniasis in an Endemic Area, of Qom Province, Central Iran. J Arthropod Borne Dis. 2017;11(3):403-13. [PubMed: 29322057]. [PubMed Central: PMC5758636].

6. Nateghi Rostami M, Saghafipour A, Vesali E. A newly emerged cutaneous leishmaniasis focus in central Iran. Int J Infect Dis. 2013;17(12):e1198-206. doi: 10.1016/j.ijid.2013.07.003. [PubMed: 24011629].

7. Ministry of Health and Medical Education (I.R.Iran) Center for Disease Management. Tehran: Principles of leishmaniasis prevention \& surveillance; 2007.4 p.

8. Salimi M, Saghafipour A, Hamidi Parsa H, Khosravi M, Shirzadi MR. Economic Burden Evaluation of Cutaneous Leishmaniasis in Iran. Shiraz E-Med J. 2019;20(6). doi: 10.5812/semj.82810.

9. Saghafipour A, Mozaffari E, Rezaei F. The evaluation of intralesional glucantime and cryotherapy plus intralesional glucantime therapeutic efficacy on zoonotic cutaneous leishmaniasis: A randomized clinical trial. Int J Pediatr. 2017;5(12):6689-97. doi: 10.22038/ijp.2017.24545.2069.

10. Azim M, Khan SA, Ullah S, Ullah S, Anjum SI. Therapeutic advances in the topical treatment of cutaneous leishmaniasis: A review. PLoS Negl Trop Dis. 2021;15(3). e0009099. doi: 10.1371/journal.pntd.0009099. [PubMed: 33657097]. [PubMed Central: PMC7928440]. 
11. Brito NC, Rabello A, Cota GF. Efficacy of pentavalent antimoniate intralesional infiltration therapy for cutaneous leishmaniasis: A systematic review. PLoS One. 2017;12(9). e0184777. doi: 10.1371/journal.pone.0184777. [PubMed: 28926630]. [PubMed Central: PMC5604971].

12. Jaffary F, Nilforoushzadeh MA, Abdellahi L, Tahmasebi Poor H. Antimonial treatment failure rate in patients with cutaneous leishmaniasis. Tehran Univ Med J TUMS Publ. 2018;76(3):197-203.

13. Desjeux P. The increase in risk factors for leishmaniasis worldwide. Transact Royal Soc Trop Med Hygiene. 2001;95(3):239-43. doi: 10.1016/s0035-9203(01)90223-8.

14. Wolf Nassif P, D. E. Mello TFP, Navasconi TR, Mota CA, Demarchi IG, Aristides SMA, et al. Safety and efficacy of current alternatives in the topical treatment of cutaneous leishmaniasis: a systematic review. Parasitology. 2017;144(8):995-1004. doi: 10.1017/S0031182017000385. [PubMed: 28367792].
15. Yazdanpanah MJ, Ebrahimirad M, Khazaeinejad S. Comparison between intralesional glucantime injection and cryotherapy in papular cutaneous leishmaniasis. J Gorgan Univ Med Sci. 2006;8(3):16-9.

16. Negera E, Gadisa E, Hussein J, Engers H, Kuru T, Gedamu L, et al. Treatment response of cutaneous leishmaniasis due to Leishmania aethiopica to cryotherapy and generic sodium stibogluconate from patients in Silti, Ethiopia. Trans R Soc Trop Med Hyg. 2012;106(8):496503. doi: 10.1016/j.trstmh.2012.02.006. [PubMed: 22503475].

17. Minodier P, Parola P. Cutaneous leishmaniasis treatment. Travel Med Infect Dis. 2007;5(3):150-8. doi:10.1016/j.tmaid.2006.09.004. [PubMed: 17448941].

18. de Menezes JP, Saraiva EM, da Rocha-Azevedo B. The site of the bite: Leishmania interaction with macrophages, neutrophils and the extracellular matrix in the dermis. Parasit Vectors. 2016;9:264 doi: 10.1186/s13071-016-1540-3. [PubMed: 27146515]. [PubMed Central: PMC4857439]. 